
\title{
28 Research Soure \\ Temporal Lobe Activation Predicts Episodic Memory Following Traumatic Brain Injury
}

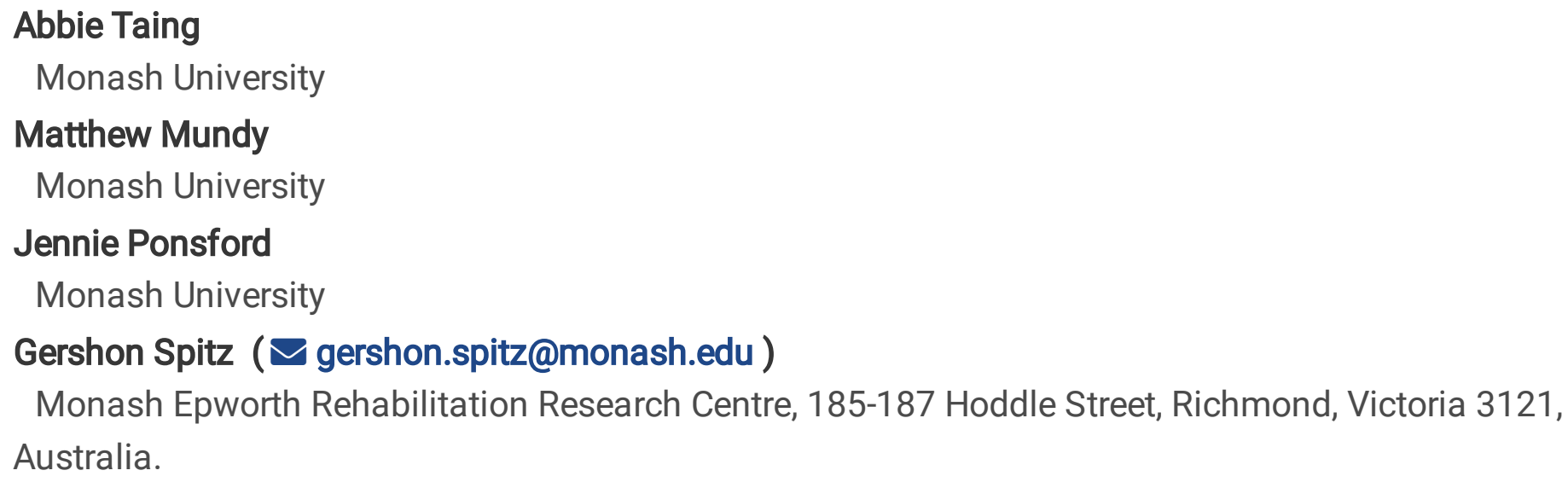

\section{Research Article}

Keywords: traumatic brain injury, episodic memory, MRI, temporal lobes

Posted Date: January 28th, 2021

DOI: https://doi.org/10.21203/rs.3.rs-152808/v1

License: (c) (1) This work is licensed under a Creative Commons Attribution 4.0 International License. Read Full License 


\section{Abstract}

The temporal lobes are critical for episodic memories and are preferentially affected following a traumatic brain injury (TBI). As such, episodic memory difficulties are common following TBI; however, the underlying neural changes that precipitate or maintain these difficulties in the early phase of recovery remains poorly understood. Here, we use functional magnetic resonance imaging (fMRI) to interrogate the relationship of temporal lobe activation in response to face, scene, and animal stimuli. Twenty-five patients with moderate to severe TBI were recruited an average of 2 months' post-injury and compared with 21 demographically similar healthy controls. Findings indicate that memory for faces was preferentially impaired, compared to scene and animal stimuli. Decreased activity in temporal lobe structures was present for both face (right transverse temporal gyrus) and scene stimuli (right fusiform gyrus), but not for animals. Greater activation in these structures was associated with better long-term recognition. These findings provide evidence to suggest that TBI: a) preferentially affects memory for complex stimuli such as faces and scenes, and b) causes aberrant neuronal processes despite lack of evidence of significant impairment in behavioural performance. The mechanisms underpinning these findings are discussed in terms of differences in verbalisation during encoding and reduced neural efficiency.

\section{Introduction}

Traumatic brain injury (TBI) can cause focal and diffuse disruption to multiple brain systems. Pathology is most widely observed in frontal and temporal cortices ${ }^{1}$, and as such impairments in processing speed, attention, executive function, and memory are most common ${ }^{2,3}$. Invariably, all individuals with a moderate or severe TBI will experience an initial transient period of impaired consciousness with amnesia and general confusion known as post-traumatic amnesia (PTA) ${ }^{4}$. Although most individuals emerge from PTA, many report ongoing difficulty with so-called episodic memories ${ }^{5,6}$. Episodic memories involve the ability to learn, store, and retrieve information about personal experiences ${ }^{7,8}$. Impairment of episodic memory can interfere with crucial skills such as new learning and task completion, and therefore can significantly limit functional independence and productivity ${ }^{9}$.

Neuroanatomically, episodic memory is supported by a brain network spanning several regions in the frontal and parietal cortices ${ }^{10,11}$. However, structures contained within the temporal lobes are arguably the most crucial for particular aspects of episodic memory 7,12 . The temporal lobes house the hippocampus, and the entorhinal, perirhinal, and parahippocampal cortices - collectively known as the medial temporal lobes ${ }^{13,14}$. The hippocampus and other medial temporal structures are involved in the encoding of episodic stimuli, and modulation of activity in these regions has been found to predict subsequent recollection of the encoded stimuli ${ }^{15}$.

The temporal lobes are preferentially affected after $\mathrm{TBI}^{1}$. The location of the temporal lobes and their proximity to the middle cranial fossa makes them highly susceptible to acceleration-deceleration forces 
present during injury ${ }^{16,17}$. Structures within the temporal lobes, such as the hippocampus and other limbic structures, are particularly susceptible to hypoxic insults and excitotoxicity effects ${ }^{12}$. As such, atrophy in the hippocampus and fornix is common following $\mathrm{TBI}^{18}$, and has been associated with impaired memory ${ }^{19}$.

Functional imaging studies in the TBI population have also implicated the temporal lobes in impaired episodic memory. Individuals with TBI tend to display increased activity in various regions, including the temporal lobes, during encoding of episodic stimuli when compared with healthy controls ${ }^{20-22}$. One limitation of these previous studies is that most have failed to use paradigms that may reflect the type of episodic memory deficits that individuals with TBI may experience in their daily life. Both Russell et al. and Arenth et al. used a mixture of line drawings of pictures and shapes, words, and letter strings, and only short-term retention was assessed ${ }^{20,21}$. Gillis and Hampstead addressed these methodological shortcomings by using realistic images of common objects and assessed long-term retention outside of the scanner ${ }^{22}$. However, participants recruited into their study were in the chronic stages of recover (range 1 to 13 years). Characterisation of memory for ecologically relevant episodic stimuli following TBI is lacking for the early period of recovery.

Here, we rectify this gap by investigating the extent to which memory for common stimuli is impaired following TBI, focussing specifically on the temporal lobe. Processing of common stimuli such as faces, scenes, and animals has been found to recruit specialised temporal lobe structures ${ }^{23,24}$. Exposure to faces robustly activates regions in the middle fusiform gyrus ('fusiform face area'), the lateral inferior occipital gyrus ('occipital face area'), and right superior temporal sulcus ${ }^{25-27}$; exposure to scenes activates the posterior parahippocampus (parahippocampal place area) ${ }^{28}$; and exposure to animals recruits activity in the bilateral fusiform gyrus ${ }^{23,29}$. Although some degree of impairment is expected given the high prevalence of temporal pathology and specialised processing of various stimuli in this area, it is possible that not all stimuli are equally impaired following injury. Past studies of amnestic patients have demonstrated stimulus-sensitive impairments for complex stimuli such as faces and scenes ${ }^{30,31}$. In the TBI population, impairment of face recognition has also been previously documented ${ }^{32}$.

The temporal lobes play a critical role in episodic memory, which is frequently impaired following TBI. Therefore, the present study specifically focusses on the temporal lobes to examine how episodic memory impairments may affect aspects of everyday memory in the early phase of recovery. We used an $\mathrm{fMRI}$ task to measure temporal lobe processing during encoding of three stimuli of varying complexity: faces, scenes, and animals. Recognition memory recognition was subsequently probed in an out-ofscanner behavioural task. In line with previous studies ${ }^{20-22}$, we hypothesised that the TBI group would display greater temporal lobe activation during stimulus encoding compared to healthy controls. Furthermore, we hypothesised that greater activity during encoding would be associated with poorer recognition task performance. Lastly, we hypothesised that individuals with TBI would be most impaired for complex stimuli such as faces and scenes. 


\section{Materials And Methods}

\section{Participants}

Twenty-five patients (17 males, 8 females) who had sustained moderate to severe TBI, determined prospectively using the Westmead Post Traumatic Amnesia Scale ${ }^{33}$, were recruited from the Acquired Brain Injury Ward at Epworth Hospital (Richmond, Victoria) after emerging from post-traumatic amnesia $(M=2.16$ months, $S D=1.48$ months, range $=0.69-6.64$ months; Table 1 and Supplementary Table S1). TBI patients predominantly had prefrontal and temporal pathology (Fig. 1). Exclusion criteria included age $<18$ or $>75$ years, prior history of TBI or other neurological conditions, significant psychiatric or substance abuse history, and MRI contraindication. Twenty-one healthy controls ( 13 males, 8 females) of similar age, sex, and education were also recruited (Table 1). There were no significant group differences on any of the demographic variables $(P>0.05)$. Due to a technical error during data collection, a subset of the sample (6 TBls and 8 healthy controls) was excluded from the MRI analyses due to poor coverage of the temporal lobes. Additionally, 1 healthy control participant was excluded due to excessive movement in the scanner. Despite this, it should be noted that our fMRI sample size is still comparable to previous neuroimaging studies ${ }^{20-22}$ of episodic memory in the TBI population. Written informed consent was provided by all participants. This study was approved by Monash Health Human Research Ethics Committee and conducted in accordance to the Declaration of Helsinki.

\section{Episodic memory paradigm}

The episodic memory paradigm is a task adapted from Mundy et al. to probe episodic memory encoding

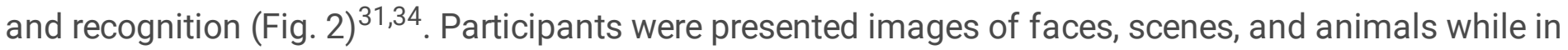
the scanner. They were instructed to respond, using trigger buttons, to each stimulus based on set criteria to ensure attention was maintained throughout the task (e.g. decide whether an animal is shorter or taller than a human man; whether the face is male or female; whether a scene looks hot or cold). consisted of six blocks, each block comprising 20 images from the three stimulus categories. This task comprised 60 unique stimuli, each presented twice throughout the session. Each stimulus was presented for 3 seconds, followed by a 3 second inter-stimulus interval. Five rest blocks were presented after the $1^{\text {st }}-5^{\text {th }}$ experimental blocks.

Memory of the stimuli presented in the fMRI task was probed in an out of scanner task. Recognition of the episodic stimuli was assessed by instructing participants to classify stimuli as "old" (i.e. images seen during the fMRI task) or "new" (i.e. images that were not presented during the fMRI task). Participants rated a total of 60 old and 60 new stimuli, each presented twice ( 240 stimuli in total). To assess retrieval 'confidence', images were rated on a scale from "definitely old" to "definitely new". Responses were considered correct/incorrect regardless of confidence level (secondary analysis indicated no significant differences in confidence rating between groups - see "Confidence rating analysis" in Supplementary for further detail). Performance for this task was assessed using accuracy and reaction times.

\section{MRI acquisition}


Structural and functional MR images were acquired in two clinical scanners, using 3.0 Tesla Siemens Magnetom Skyra scanners and a 20-channel head coils. Functional images were acquired using singleshot gradient-echo planar imaging (EPI) with the following parameters: repetition time $(T R)=2.75 \mathrm{~s}$; echo time $(\mathrm{TE})=30 \mathrm{~ms}$; flip angle $=90^{\circ} ; 220 \times 220$ matrix; voxel size $=3.4 \times 3.4 \times 3.0 \mathrm{~mm}$. A high-resolution 3D T1-weighted image covering the entire brain was also acquired for anatomical reference $(T R=2.3 \mathrm{~s} ; \mathrm{TE}=$ $2.32 \mathrm{~ms}$; flip angle $=8^{\circ} ; 236 \times 350$ matrix; voxel size $\left.=0.9 \times 0.9 \times 0.9 \mathrm{~mm}\right)$. Due to reduced brain coverage (due to using clinical scanners), and a-priori hypotheses, we focused on the temporal lobes.

\section{Statistical analysis}

\section{Behavioural and demographic data}

Behavioural and demographic data were analysed using $R$ version 3.6.0 (R Core Team, 2019). Two-tailed independent samples t-tests were used to examine group differences on the demographic variables including age, sex, and years of education. Behavioural data were screened for normality, transformed (if necessary), and assessed for violation of statistical assumptions prior to analysis. Outcome measures were analysed using linear mixed models to account for clustering or non-independence of measures within participants. Memory performance was assessed using dprime and reaction time. Dprime measured task accuracy, accounting for the signal (hits) and noise (false alarms). Reaction times were generated by obtaining the average reaction time per stimulus category. Task accuracy was assessed by modelling stimulus category, group, and their interaction (stimulus category $\mathrm{x}$ group) as fixed effects, and participant as a random effect. For reaction time, the data were inversely transformed, and a model was fitted with stimulus category, group, and an interaction (stimulus category $x$ group) as fixed effects, and participant as a random effect. Age and education were also added as covariates in the models, given their influence on memory performance ${ }^{35,36}$ and reaction time ${ }^{37}$. Where appropriate, post-hoc analyses were conducted using two-tailed t-tests multiple comparison correction.

\section{Imaging data}

\section{MRI preprocessing}

Prior to preprocessing, lesions were manually segmented using MRIcron (http://www.mricro.com/mricron). Preprocessing was performed using fMRIPrep 20.0.0 (Esteban et al., 2018) and involved the application of the following step: undistortion of EPI data, realignment, normalisation, and estimation of confounds. Further information about the MRI preprocessing can be accessed in the Supplementary (see "Detailed MRI preprocessing").

\section{fMRI analysis}

fMRI data were analysed using FSL's FEAT version 6.0.2 (FMRIB's Software Library, www.fmrib.ox.ac.uk/fsl). In the first level analysis, contrasts between each stimulus category (i.e. animals, faces, scenes) and the rest blocks were generated for each participant. The onset times for each 
contrast corresponded to the first stimulus presentation of each category and were 27 seconds in duration. To reduce motion-related artifacts, additional regressors using a modified method of the anatomical CompCor that explained $50 \%$ of the variance were also included in the first level model ${ }^{38}$. Differences in brain activation of categories of episodic stimuli were assessed in a 2 (group: TBI vs. healthy controls) $\times 3$ (stimulus category: faces, scenes, and animals) factorial design using FLAME 1+2 mixed effects with automatic outlier de-weighting. An additional explanatory variable was also added at the group level to control for the acquisition of images from two scanners. Given our hypothesis regarding structures in the temporal lobes and their role in learning and processing of category-specific stimuli, an a-priori region of interest (ROI) mask of the temporal lobe was generated using the MNI Structural Atlas (Supplementary Fig. S1) and used in the group level analysis. Imaging findings are reported using a cluster level threshold of $Z>3.1$ and a family wise error cluster correction threshold of $P$ $<0.05$. FEAT contrast of parameter estimates (COPE) were extracted from significant clusters at the group level. To investigate differences in BOLD response, two-tailed independent samples t-tests were conducted using COPE values. Finally, association between BOLD response and behavioural performance on the episodic recognition task were assessed using Pearson correlations.

\section{Results}

\section{Episodic memory is selectively impaired for face stimuli}

First, we investigated whether recognition of episodic memories was impaired following TBI. To do this, we performed a linear mixed model assessing memory recall accuracy (old vs. new, as measured using dprime) on the episodic retrieval task. Overall, the TBI group demonstrated significantly poorer recognition accuracy than healthy controls $(95 \% \mathrm{Cl}, 0.04-0.72 ; P=0.028 ; \mathrm{Fig}$. 3). Post-hoc analyses indicated that the group difference was driven by a significant difference in accuracy for faces $(95 \% \mathrm{Cl}$, $1.11-1.50 ; P=0.037)$. There was a trend for lower accuracy for scenes, however, this did not reach statistical significance $(95 \% \mathrm{Cl}, 0.94-1.19 ; P=0.169)$. There was no significant difference between the groups in accuracy for animals $(95 \% \mathrm{Cl}, 1.12-1.26 ; P=0.440)$.

\section{Reaction times were quickest for face stimuli}

Next, we investigated whether reaction time was significantly different between the groups and whether it varied according to the type of stimulus. Overall, reaction time was greater for individuals with TBI compared to healthy controls, irrespective of stimulus type $(95 \% \mathrm{Cl}, 0.08-0.25 ; P<0.001 ;$ Fig. 3). Posthoc analyses revealed that the TBI group was significantly slower than healthy controls in responding to faces $(95 \% \mathrm{Cl}, 0.48-0.64 ; P<0.001)$, scenes $(95 \% \mathrm{Cl}, 0.43-0.58 ; P=0.001)$, and animals $(95 \% \mathrm{Cl}, 0.42$ $-0.58 ; P=0.001)$. Across both groups, reaction time also varied depending on the stimulus category: the TBI group was quicker to respond to faces than scenes $(95 \% \mathrm{Cl}, 0.43-0.48 ; P=0.014)$ and animals ( $95 \%$ $\mathrm{Cl}, 0.42-0.48 ; P=0.005)$; similarly, healthy controls were quicker to respond to faces than scenes ( $95 \%$ $\mathrm{Cl}, 0.58-0.64 ; P=0.003)$ and animals $(95 \% \mathrm{Cl}, 0.58-0.64 ; P=0.001)$. Given this pattern of results, we further investigated whether the poorer performance for faces in the TBI group was driven by a speed- 
accuracy trade-off. To do this, we included reaction time as a covariate in the linear mixed model. Results indicated that participants with TBI still performed significantly poorer than healthy controls after controlling for reaction time $(95 \% \mathrm{Cl}, 1.10-1.54 ; P=0.027)$, suggesting that this pattern of performance was not due solely to a speed-accuracy trade-off.

\section{fMRI Task activates the stereotypical regions underpinning encoding of episodic stimuli}

To demonstrate that our task elicited activations in stereotypical areas involved with the canonical network that support encoding of episodic stimuli, we first included all participants in an analysis looking at the average activation for each stimulus category (i.e. faces, scenes, and animals; Fig. 4). During encoding of face stimuli, significant clusters were noted in face-selective areas including the right inferior occipital gyrus and left/right fusiform gyrus ${ }^{25-27}$, as well as the right hippocampus. During encoding of scene stimuli, significant clusters were noted in scene-selective area of right parahippocampal gyrus ${ }^{28}$, as well as the left fusiform gyrus. Finally, during encoding of animal stimuli, significant clusters were noted in animal-selective area of the right fusiform gyrus ${ }^{23,29}$, as well as the left inferior occipital gyrus.

\section{TBI patients show reduced right transverse temporal gyrus activation during face processing}

Consistent with the behavioural results, group differences on imaging were apparent during encoding of faces. TBI participants showed reduced activation in the right transverse temporal gyrus extending to the planum temporale compared to healthy controls (Fig. 5).

FEAT analysis COPE values were extracted for the significant cluster. We first investigated whether there was a significant difference in BOLD response between groups using an independent samples t-test. As expected, TBI patients displayed lower COPE values $(M=-9.90, S D=24.79)$ compared to healthy controls $(M=19.07, S D=33.04), t(18)=-2.61, P=0.017$. We further examined whether there was an association with behavioural performance on the episodic recognition task using Pearson correlations. Overall, there was a moderate positive relationship between COPE values and the dprime scores for face stimuli, $r(28)=$ $0.497, P=0.005$. Follow-up correlations indicated that a significant correlation was only apparent for the TBI group, $r(16)=0.480, P=0.044$, and not healthy controls, $r(10)=0.375, P=0.229$. To examine specificity of this brain-behaviour relationship, we conducted a control analysis, correlating whole temporal lobe activity with dprime scores for face stimuli. Whole temporal lobe activation was not correlated with face recognition performance $(P>0.05$; see "Additional control analyses" in Supplementary).

\section{TBI patients display reduced right fusiform gyrus activity during scene processing}

Despite a non-significant difference between groups for scene recognition, we found reduced activation in the right posterior fusiform gyrus for the TBI group in comparison to healthy controls during scene encoding (Fig. 6). 
TBI patients showed lower COPE values $(M=29.26, S D=25.11)$ compared to controls $(M=76.93, S D=$ 47.76), $t(15)=3.18, P=0.006$. To further examine whether functional changes in this cluster were associated with behavioural performance, we again conducted a series of Pearson correlations. Upon removal of an outlier (see "Additional scene cluster results" in Supplementary for further detail), there was a moderate postive relationship between the COPE values extracted from this cluster and the dprime scores for scene stimuli, $r(27)=0.482, P=0.008$. Further examation revealed that a significant correlation was only apparent for the TBI group, $r(15)=0.574, P=0.016$, and not healthy controls, $r(10)=0.317, P=$ 0.316 . Similar to the control analysis above, we conducted a control analysis, correlating whole temporal lobe activity with dprime scores for scene stimuli. Interestingly, general temporal lobe activation was correlated with scene recognition performance $(P=0.006)$. However, correlations were no longer significant once the groups were examined separately $(P>0.05$; see "Additional control analyses" in Supplementary).

\section{Discussion}

The present study focused on determining the role of temporal lobe activity in episodic memory behaviour following TBI. We showed for the first time, using converging evidence from behavioural and fMRI data, that episodic memory impairment following TBI appeared to be category-specific and related specific sub-regions within the temporal lobes. Deficits were most apparent for faces; TBI patients displayed reduced transverse temporal gyrus activation during face encoding and subsequent impairment on face recognition. TBI patients also displayed reduced fusiform gyrus activation for scenes; this is despite no statistically significant difference between groups during scene recognition. Interestingly, brain activation during face and scene encoding correlated with subsequent recognition for TBI patients but not in healthy control participants. Overall, these findings suggest that TBI: a) preferentially impairs episodic memory in specific domains, and b) aberrant neural processing may not be reflected in statistically significant differences behavioural assessments, and thus neural activation conveys complimentary information undetected through examination of overt behaviour.

Broadly speaking, our findings are similar to those of previous studies of amnestic patients who show stimulus-sensitive impairments for complex stimuli such as faces and scenes ${ }^{30,31}$. Our findings are also in concordance with a previous study demonstrating impaired face recognition in the TBI population ${ }^{32}$. Valentine et al. subjected participants to a range of facial recognition and learning tasks and found that while performance varied, deficits were more apparent for tasks with greater demands ${ }^{32}$. More specifically, the most sensitive tasks were those which contained a larger number of faces to be encoded or had fewer presentations of the stimuli. Our task was comparably difficult in that participants were presented with a similar number of face stimuli which were only shown twice during the encoding phase; thus, it was not surprising we obtained a similar finding.

As expected, we found that the TBI group was generally slower than healthy controls in their reaction times. Both groups, however, responded more quickly to faces than to animals and scenes. This result somewhat aligns with a study conducted by Keightley et al. who found that participants reacted quicker 
to faces than scenes, despite accuracy being better for scene stimuli ${ }^{39}$. We further explored whether speed-accuracy trade off could account for our findings, given participants displayed the poorest accuracy for face stimuli. We found that including reaction time as a covariate when determining between-group differences in face accuracy did not change the result. Instead, the rapid response to faces suggests that individuals with TBI may have performed superficial encoding of face stimuli, thus negatively impacting decision-making during recognition.

A unique aspect of our study was the inclusion of animal and scene stimuli, in addition to faces, which allowed us to investigate the effects of stimulus complexity on episodic memory. Interestingly, we found the TBI group displayed altered neural activity without showing statistically significant impairment of behavioural performance for scene stimuli, whereas changes on both functional and behavioural measures were apparent for faces. One potential reason for these results is that faces are more visually complex than scenes. Indeed, facial processing is a complex phenomenon requiring multifaceted processes across widespread cortical areas ${ }^{40}$. Unlike most other visual stimuli they are processed in a holistic and configural manner 41,42 ; thus, discrimination requires attention to detail and subtle perception of variable facial features ${ }^{40}$.

Our $\mathrm{fMRI}$ results provide further insights into the mechanisms that may underlie behavioural deficits for faces. We found that the TBI group showed a reduced response in the right transverse temporal gyrus extending to the planum temporale during encoding of faces. However, these areas do not form part of the core or extended face network ${ }^{26}$. The transverse temporal gyrus is predominantly implicated in auditory processing ${ }^{43,44}$, although studies have also demonstrated its role in spontaneous inner speech $^{45}$. The planum temporale has been shown to be involved in language functions ${ }^{46}$. Therefore, it is possible that difference in BOLD activity in this temporal lobe region may reflect differences in verbalisation during face encoding. Interestingly, activation of this region correlated with behavioural performance only for individuals with TBI. It may be that individuals with TBI may therefore have made less attempts to verbally describe the stimuli when processing faces. This further supports the hypothesis that TBI participants encoded these stimuli in a rapid and superficial manner. In contrast, healthy controls did not display such an association, suggesting verbalisation during face encoding was not key in determining later recognition success.

Our other key finding was that the TBI group demonstrated reduced brain activity during encoding of scenes in the right posterior fusiform gyrus. The right posterior fusiform gyrus responds non-selectively to faces and scenes and generally may be involved with processing complex visual stimuli ${ }^{47}$. This result suggests impaired recruitment of this temporal lobe sub-region during processing of scenes. Indeed, we found that individuals with TBI who had higher activation within this region performed better during scene recognition. Despite differences in brain activation, the groups did not differ with respect to behaviour. This finding may be due to scene stimuli containing a greater number of contextual cues that could further aid encoding and recollection. For example, individuals may have used cues such as the location (e.g. kitchen) or remembered certain salient scene features (e.g. item/s contained in the scene). 
To examine the specificity of the brain-behaviour relationships, we conducted a series of control analyses to determine whether the association between brain activation and behavioural performance was specific to the significant clusters or, more generally, to activity in the temporal lobes. In support of our main findings, general activation in the temporal lobes was not associated with face recognition accuracy, thus highlighting the specificity of right transverse temporal gyrus in predicting face recognition performance. However, we found that general activation in the temporal lobes was associated scene recognition accuracy. This relationship disappeared when the TBI group and healthy controls were considered separately. This indicates that the right fusiform gyrus has less specificity in predicting scene recognition performance. In line with our behaviour findings, these results suggest greater, more robust, specificity for face stimuli, compared to scenes.

Although our findings show a clear brain-behaviour relationship, these associations oppose our initial hypotheses, which predicted that individuals with TBI would display greater temporal lobe activity in support of previous studies ${ }^{20-22}$. One general model that could explain this finding is that of cortical reorganisation following injury ${ }^{48-50}$. That is, the pattern of cortical activation reflecting neural compensation or recovery following TBI is likely to depend on the length of time since an individual's injury ${ }^{51}$. A key methodological difference is that our TBI participants were recruited at an average of 2 months' post-injury whereas those in past studies were recruited over 1 year post-injury\{Arenth, 2012 \#40\} ${ }^{20-22}$ \{Arenth, 2012 \#40\}\{Russell, 2011 \#14\}\{Arenth, 2012 \#40\}\{Arenth, 2014 \#520\}. In a key study, Sanchez-Carrion et al. characterised the longitudinal changes in brain activity following $\mathrm{TBI}^{52}$. They did so by assessing brain activity during a working memory task at 6 months and 1 year following the injury. Their findings show an initial reduction in brain activity at the 6 month time-points which gradually resolved by 1 year following the injury. Thus, discrepancies in our findings from past studies may reflect differences in recovery phases.

Our study has several important implications. From a clinical perspective, it is generally acknowledged that individuals have generalised episodic memory deficits after injury. Our findings provide evidence to the contrary and show that impairment is more apparent with complex visual stimuli such as faces and scenes. An obvious clinical translation is the need to provide strategies that promote deeper processing to better aid memory for these and other complex stimuli. In addition, our study provides further support for the utility of $\mathrm{fMRI}$ as a complimentary source of information by demonstrating evidence of aberrant neural processing that was less evident in behavioural performance. This is important considering that most assessments of memory are based on behavioural performance.

There were some limitations in our study, however. Our episodic memory paradigm only allowed us to investigate functional activity during stimulus encoding. Therefore, we could not comment on the extent in which temporal structures are implicated in recognition of episodic stimuli following TBI. This may be an avenue for exploration in future research. Although not a limitation per se, we took an a-priori approach specifically focusing on the temporal lobes. While this allowed us to answer specific questions about the temporal lobes' role in processing of episodic stimuli, it also limited our investigation of non- 
temporal contributions to episodic memory. For example, there is value in investigating the interaction between frontal and temporal regions to support encoding of more complex stimuli, especially considering the frontal lobes' role in strategy, allocation of resources, and planning ${ }^{53,54}$.

In conclusion, we found evidence demonstrating that individuals with TBI show impairment of episodic memory for complex stimuli and that this was associated with functional changes. In comparison to healthy controls, we found that the TBI group displayed reduced activation in the right transverse temporal gyrus and fusiform gyrus during face and scene processing, respectively. We found that brain activation in these temporal lobe sub-regions were associated with behavioural performance for the TBI group and not healthy controls. These findings may be explained in terms of differences in verbalisation during encoding and cortical reorganisation. Overall, we provide preliminary evidence demonstrating that following TBI: a) episodic memory impairment is domain specific and more broadly dependent on the complexity of the stimuli, and b) aberrant neuronal activity may exist despite lack of evidence of significant impairment in behavioural performance, and therefore neural activation may be a more robust early indicator than behaviour.

\section{Abbreviations}

$\mathrm{TBI}=$ traumatic brain injury; functional magnetic resonance imaging = $\mathrm{fMRI}$; $\mathrm{GSC}=$ Glasgow Coma Scale; Post Traumatic Amnesia = PTA; WPTAS = Westmead Post Traumatic Amnesia Scale; DAl $=$ diffuse axonal injury; $\mathrm{EDH}$ = extradural haematoma; $\mathrm{ICH}$ = intracerebral haemorrhage; $\mathrm{SAH}=$ subarachnoid haemorrhage; $\mathrm{SDH}=$ subdural haemorrhage; $\mathrm{NAD}=$ no abnormality detected; $\mathrm{TR}=$ repetition time; $\mathrm{TE}=$ echo time; contrast of parameter estimates $=$ COPE

\section{Declarations}

\section{Acknowledgements}

GS was funded by a National Health and Research Council Early Career Fellowship (APP1104692) and the Brain Foundation. This work was supported by the Multi-modal Australian ScienceS Imaging and Visualisation Environment (MASSIVE) HPC facility (www.massive.org.au). In addition, the authors would like to thank the staff at the Acquired Brain Injury Ward at Epworth Hospital (Richmond) and Bridge Road Imaging. The authors would also like to thank the participants who took part in the study.

\section{Authors contribution}

AT collected/analysed the data and wrote the manuscript; MM conceptualised the study, designed the study, and reviewed the manuscript; JP conceptualised the study and reviewed the manuscript; GS conceptualised the study, designed the study, collected/analysed the data, and reviewed the manuscript.

\section{Ethics declaration}




\section{Completing interests}

The authors declare no competing interests.

\section{Data availability}

All data supporting the findings of this study can be requested from the corresponding author.

\section{References}

1 Bigler, E. D. The lesion (s) in traumatic brain injury: Implications for clinical neuropsychology. Archives of clinical neuropsychology16, 95-131 (2001).

2 Draper, K. \& Ponsford, J. Cognitive functioning ten years following traumatic brain injury and rehabilitation. Neuropsychology22, 618-625, doi:10.1037/0894-4105.22.5.618 (2008).

3 Azouvi, P., Arnould, A., Dromer, E. \& Vallat-Azouvi, C. Neuropsychology of traumatic brain injury: An expert overview. Rev Neurol (Paris)173, 461-472, doi:10.1016/j.neurol.2017.07.006 (2017).

4 Tittle, A. \& Burgess, G. H. Relative contribution of attention and memory toward disorientation or posttraumatic amnesia in an acute brain injury sample. Brain Injury 25, 933-942, doi:10.3109/02699052.2011.597042 (2011).

5 Rabinowitz, A. R. \& Levin, H. S. Cognitive sequelae of traumatic brain injury. Psychiatr Clin North Am37, 1-11, doi:10.1016/j.psc.2013.11.004 (2014).

6 Vakil, E. The effect of moderate to severe traumatic brain injury (TBI) on different aspects of memory: a selective review. J Clin Exp Neuropsycho/27, 977-1021, doi:10.1080/13803390490919245 (2005).

7 Moscovitch, M., Cabeza, R., Winocur, G. \& Nadel, L. Episodic Memory and Beyond: The Hippocampus and Neocortex in Transformation. Annu Rev Psycho/67, 105-134, doi:10.1146/annurev-psych-113011143733 (2016).

8 Tulving, E. Episodic memory: from mind to brain. Annual review of psychology53, 1-25 (2002).

9 Nakase-Richardson, R. et al. Utility of post-traumatic amnesia in predicting 1-year productivity following traumatic brain injury: comparison of the Russell and Mississippi PTA classification intervals. J Neurol Neurosurg Psychiatry82, 494-499, doi:10.1136/jnnp.2010.222489 (2011).

10 Wagner, A. D., Shannon, B. J., Kahn, I. \& Buckner, R. L. Parietal lobe contributions to episodic memory retrieval. Trends Cogn Sci9, 445-453, doi:10.1016/j.tics.2005.07.001 (2005).

11 Eichenbaum, H. Prefrontal-hippocampal interactions in episodic memory. Nat Rev Neurosci18, 547558, doi:10.1038/nrn.2017.74 (2017). 
12 Dickerson, B. C. \& Eichenbaum, H. The episodic memory system: neurocircuitry and disorders. Neuropsychopharmacology35, 86-104, doi:10.1038/npp.2009.126 (2010).

13 Simons, J. S. \& Spiers, H. J. Prefrontal and medial temporal lobe interactions in long-term memory. Nat Rev Neurosci4, 637-648, doi:10.1038/nrn1178 (2003).

14 Graham, K. S., Barense, M. D. \& Lee, A. C. Going beyond LTM in the MTL: a synthesis of neuropsychological and neuroimaging findings on the role of the medial temporal lobe in memory and perception. Neuropsychologia48, 831-853, doi:10.1016/j.neuropsychologia.2010.01.001 (2010).

15 Cameron, K. A., Yashar, S., Wilson, C. L. \& Fried, I. Human hippocampal neurons predict how well word pairs will be remembered. Neuron30, 289-298 (2001).

16 Barlow, K. M. Traumatic brain injury. Handb Clin Neuro/112, 891-904, doi:10.1016/B978-0-444-529107.00011-8 (2013).

17 Daneshvar, D. H. \& McKee, A. C. Traumatic Brain Injury. 219-235, doi:10.1016/b978-0-12-3982704.00016-1 (2015).

18 Bigler, E. D. et al. Traumatic brain injury and memory: The role of hippocampal atrophy. Neuropsychology10, 333 (1996).

19 Ariza, M. et al. Hippocampal head atrophy after traumatic brain injury. Neuropsychologia44, 19561961, doi:10.1016/j.neuropsychologia.2005.11.007 (2006).

20 Russell, K. C., Arenth, P. M., Scanlon, J. M., Kessler, L. J. \& Ricker, J. H. A functional magnetic resonance imaging investigation of episodic memory after traumatic brain injury. J Clin Exp Neuropsycho/33, 538547, doi:10.1080/13803395.2010.537253 (2011).

21 Arenth, P. M., Russell, K. C., Scanlon, J. M., Kessler, L. J. \& Ricker, J. H. Encoding and recognition after traumatic brain injury: neuropsychological and functional magnetic resonance imaging findings. $J$ Clin Exp Neuropsycho/34, 333-344, doi:10.1080/13803395.2011.633896 (2012).

22 Gillis, M. M. \& Hampstead, B. M. A two-part preliminary investigation of encoding-related activation changes after moderate to severe traumatic brain injury: hyperactivation, repetition suppression, and the role of the prefrontal cortex. Brain Imaging Behav9, 801-820, doi:10.1007/s11682-014-9337-5 (2015).

23 Downing, P. E., Chan, A. W., Peelen, M. V., Dodds, C. M. \& Kanwisher, N. Domain specificity in visual cortex. Cereb Cortex16, 1453-1461, doi:10.1093/cercor/bhj086 (2006).

24 Mundy, M. E. et al. Material-independent and material-specific activation in functional MRI after perceptual learning. Neuroreport20, 1397-1401 (2009). 
25 Hoffman, E. A. \& Haxby, J. V. Distinct representations of eye gaze and identity in the distributed human neural system for face perception. Nature neuroscience3, 80-84 (2000).

26 Haxby, J. V., Hoffman, E. A. \& Gobbini, M. I. Human neural systems for face recognition and social communication. Biological psychiatry51, 59-67 (2002).

27 Kesler, M. L. et al. Neural substrates of facial emotion processing using fMRI. Cognitive Brain Research11, 213-226 (2001).

28 Epstein, R. A. \& Ward, E. J. How reliable are visual context effects in the parahippocampal place area? Cereb Cortex20, 294-303, doi:10.1093/cercor/bhp099 (2010).

29 Rogers, T. T., Hocking, J., Mechelli, A., Patterson, K. \& Price, C. Fusiform activation to animals is driven by the process, not the stimulus. Journal of Cognitive Neuroscience17, 434-445 (2005).

30 Taylor, K. J., Henson, R. N. \& Graham, K. S. Recognition memory for faces and scenes in amnesia: dissociable roles of medial temporal lobe structures. Neuropsychologia45, 2428-2438, doi:10.1016/j.neuropsychologia.2007.04.004 (2007).

31 Mundy, M. E., Downing, P. E., Dwyer, D. M., Honey, R. C. \& Graham, K. S. A critical role for the hippocampus and perirhinal cortex in perceptual learning of scenes and faces: complementary findings from amnesia and FMRI. Journal of Neuroscience33, 10490-10502 (2013).

32 Valentine, T., Powell, J., Davidoff, J., Letson, S. \& Greenwood, R. Prevalence and correlates of face recognition impairments after acquired brain injury. Neuropsychol Rehabil16, 272-297, doi:10.1080/09602010500176443 (2006).

33 Shores, E. A., Marosszeky, J., Sandanam, J. \& Batchelor, J. Preliminary validation of a clinical scale for measuring the duration of post-traumatic amnesia. Med J Aust144, 569-572 (1986).

34 Mundy, M. E., Downing, P. E. \& Graham, K. S. Extrastriate cortex and medial temporal lobe regions respond differentially to visual feature overlap within preferred stimulus category. Neuropsychologia50, 3053-3061 (2012).

35 Hoyer, W. J. \& Verhaeghen, P. in Handbook of the psychology of aging 209-232 (Elsevier, 2006).

36 Lachman, M. E., Agrigoroaei, S., Murphy, C. \& Tun, P. A. Frequent cognitive activity compensates for education differences in episodic memory. The American Journal of Geriatric Psychiatry18, 4-10 (2010).

37 Der, G. \& Deary, I. J. The relationship between intelligence and reaction time varies with age: Results from three representative narrow-age age cohorts at 30, 50 and 69 years. Intelligence64, 89-97 (2017).

38 Muschelli, J. et al. Reduction of motion-related artifacts in resting state fMRI using aCompCor. Neuroimage96, 22-35, doi:10.1016/j.neuroimage.2014.03.028 (2014). 
39 Keightley, M. L., Chiew, K. S., Anderson, J. A. \& Grady, C. L. Neural correlates of recognition memory for emotional faces and scenes. Soc Cogn Affect Neurosci6, 24-37, doi:10.1093/scan/nsq003 (2011).

40 Haxby, J. V., Hoffman, E. A. \& Gobbini, M. I. The distributed human neural system for face perception. Trends Cogn. Sci.4, 223-233 (2000).

41 Park, J., Newman, L. I. \& Polk, T. A. Face processing: the interplay of nature and nurture. The Neuroscientist15, 445-449 (2009).

42 Maurer, D., Le Grand, R. \& Mondloch, C. J. The many faces of configural processing. Trends Cogn. Sci.6, 255-260 (2002).

43 Kaas, J. H., Hackett, T. A. \& Tramo, M. J. Auditory processing in primate cerebral cortex. Curr. Opin. Neurobiol.9, 164-170 (1999).

44 Warrier, C. et al. Relating structure to function: Heschl's gyrus and acoustic processing. J. Neurosci.29, 61-69 (2009).

45 Hurlburt, R. T., Alderson-Day, B., Kühn, S. \& Fernyhough, C. Exploring the ecological validity of thinking on demand: neural correlates of elicited vs. spontaneously occurring inner speech. PloS one11, e0147932 (2016).

46 Shapleske, J., Rossell, S. L., Woodruff, P. \& David, A. The planum temporale: a systematic, quantitative review of its structural, functional and clinical significance. Brain Research Reviews29, 26-49 (1999).

47 Nakamura, K. et al. Functional delineation of the human occipito-temporal areas related to face and scene processing: a PET study. Brain123, 1903-1912 (2000).

48 Levine, B. et al. Functional reorganisation of memory after traumatic brain injury: a study with H2150 positron emission tomography. Journal of Neurology, Neurosurgery \& Psychiatry73, 173-181 (2002).

49 Christodoulou, C. et al. Functional magnetic resonance imaging of working memory impairment after traumatic brain injury. Journal of Neurology, Neurosurgery \& Psychiatry71, 161-168 (2001).

50 Hillary, F. G. Neuroimaging of working memory dysfunction and the dilemma with brain reorganization hypotheses. J Int Neuropsychol Soc14, 526-534, doi:10.1017/S1355617708080788 (2008).

51 Munoz-Cespedes, J. M., Rios-Lago, M., Paul, N. \& Maestu, F. Functional neuroimaging studies of cognitive recovery after acquired brain damage in adults. Neuropsychol Rev15, 169-183, doi:10.1007/s11065-005-9178-5 (2005).

52 Sanchez-Carrion, R. et al. A longitudinal fMRI study of working memory in severe TBI patients with diffuse axonal injury. Neuroimage43, 421-429, doi:10.1016/j.neuroimage.2008.08.003 (2008). 
53 Vakil, E., Greenstein, Y., Weiss, I. \& Shtein, S. The Effects of Moderate-to-Severe Traumatic Brain Injury on Episodic Memory: a Meta-Analysis. Neuropsychol Rev29, 270-287, doi:10.1007/s11065-019-09413-8 (2019).

54 Stuss, D. T. \& Alexander, M. P. Does damage to the frontal lobes produce impairment in memory? Current Directions in Psychological Science14, 84-88 (2005).

\section{Tables}

Table 1. Demographic information and clinical characteristics of the TBI and healthy groups.

\begin{tabular}{|lll|}
\hline Demographic variables & $\begin{array}{l}\text { Traumatic brain injury } \\
\text { Mean (SD) }\end{array}$ & $\begin{array}{l}\text { Healthy controls, } \\
\text { Mean (SD) }\end{array}$ \\
\hline Age (years) & $38.36(16.82)$ & $37.29(17.89)$ \\
\hline Sex (male/female) & $17 / 8$ & $13 / 8$ \\
\hline Education (years) & $13.82(3.27)$ & $14.19(2.57)$ \\
\hline Time since injury (months) & $2.16(1.51)$ & - \\
\hline PTA (days) & $22.46(14.63)$ & - \\
\hline GCS (lowest) & $9(4.37)$ & - \\
\hline
\end{tabular}

GSC = Glasgow Coma Scale; PTA = post-traumatic amnesia. Note: PTA duration were available for $n=24$ patients.

\section{Figures}

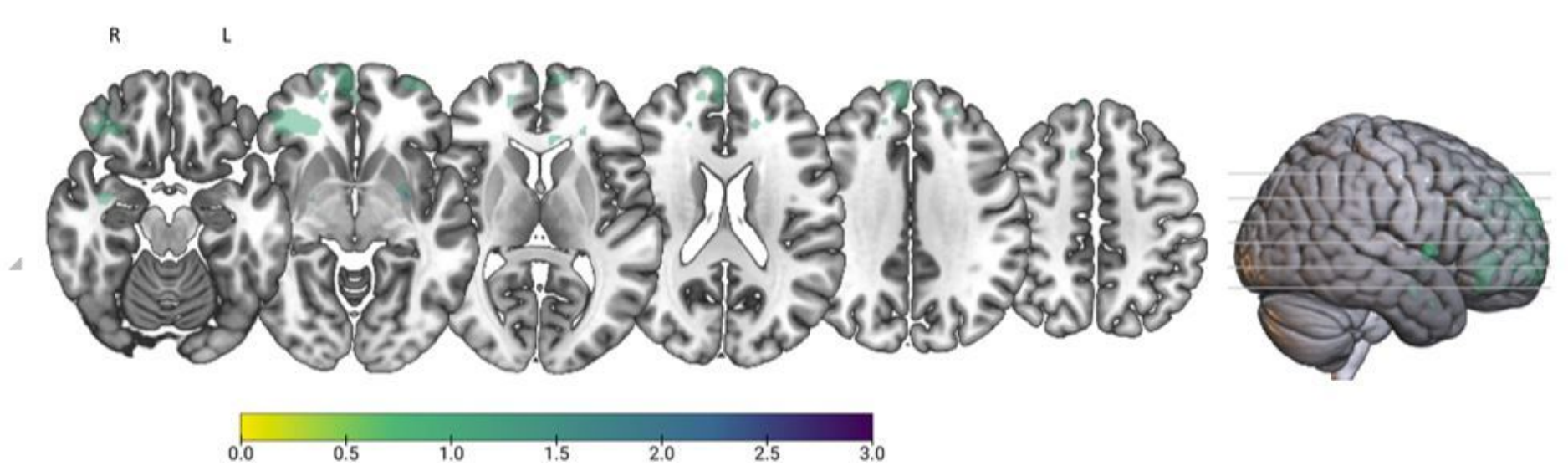

Figure 1 
Lesion overlay plot of all TBI participants. Maps were overlaid on a T1 template in MNI space.

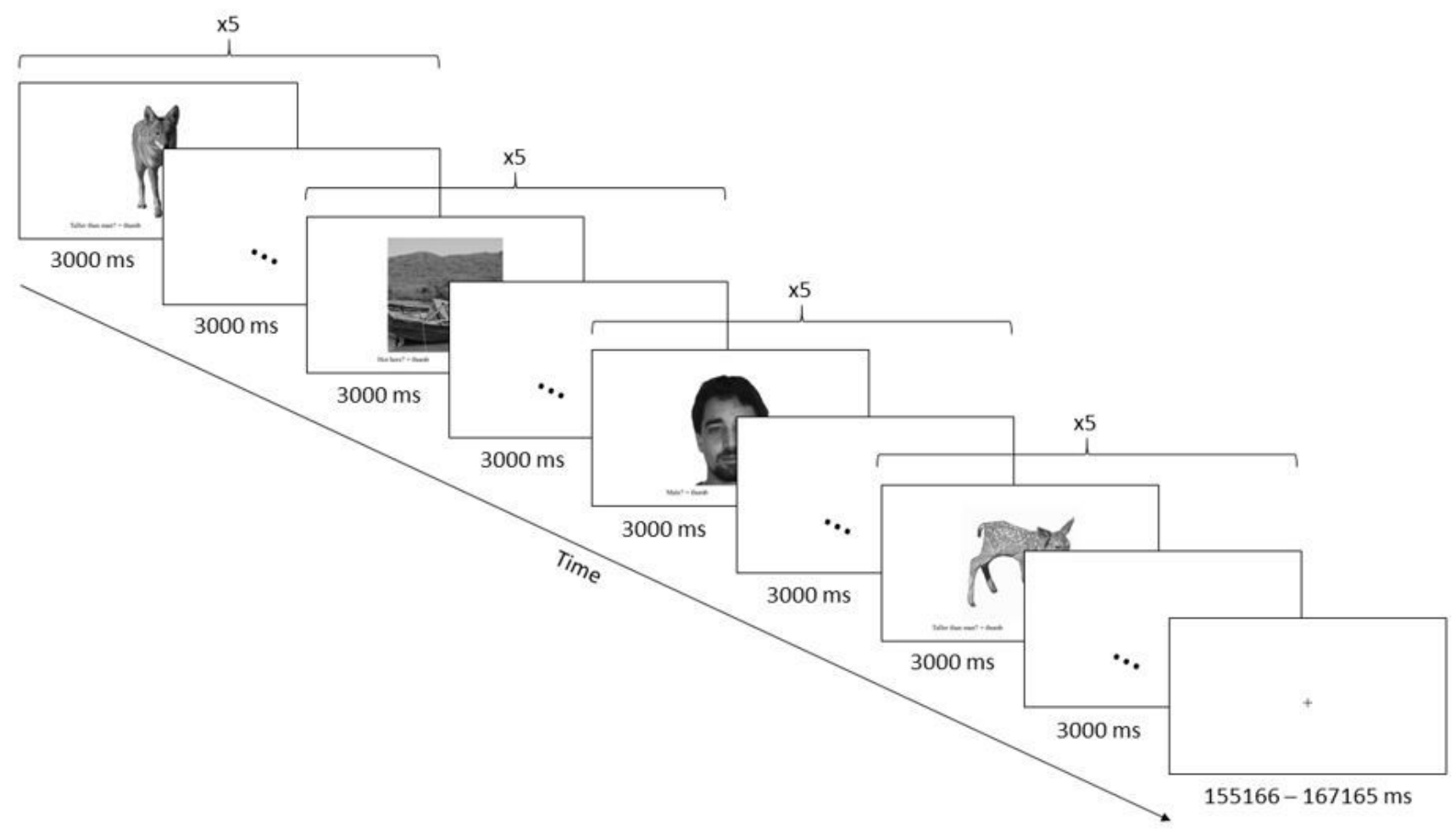

\section{Figure 2}

Schematic diagram of the episodic encoding task. Participants were presented 5 images from a stimulus category (i.e. faces, scenes, and animals) and were instructed to response to the various stimuli on screen based on set criteria. Each stimulus was presented for $3 \mathrm{~s}$, followed by an inter-stimulus duration of $3 \mathrm{~s}$. 

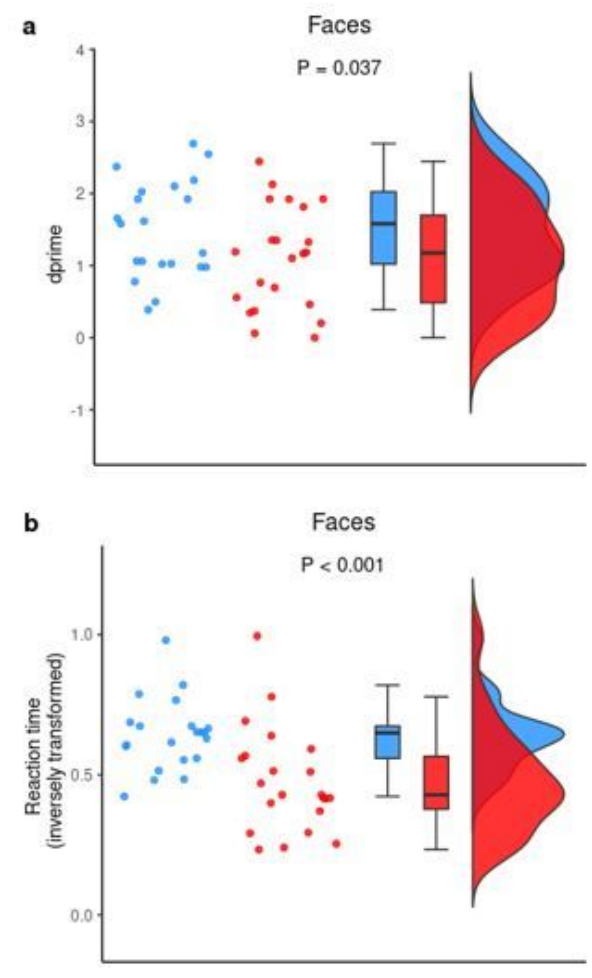
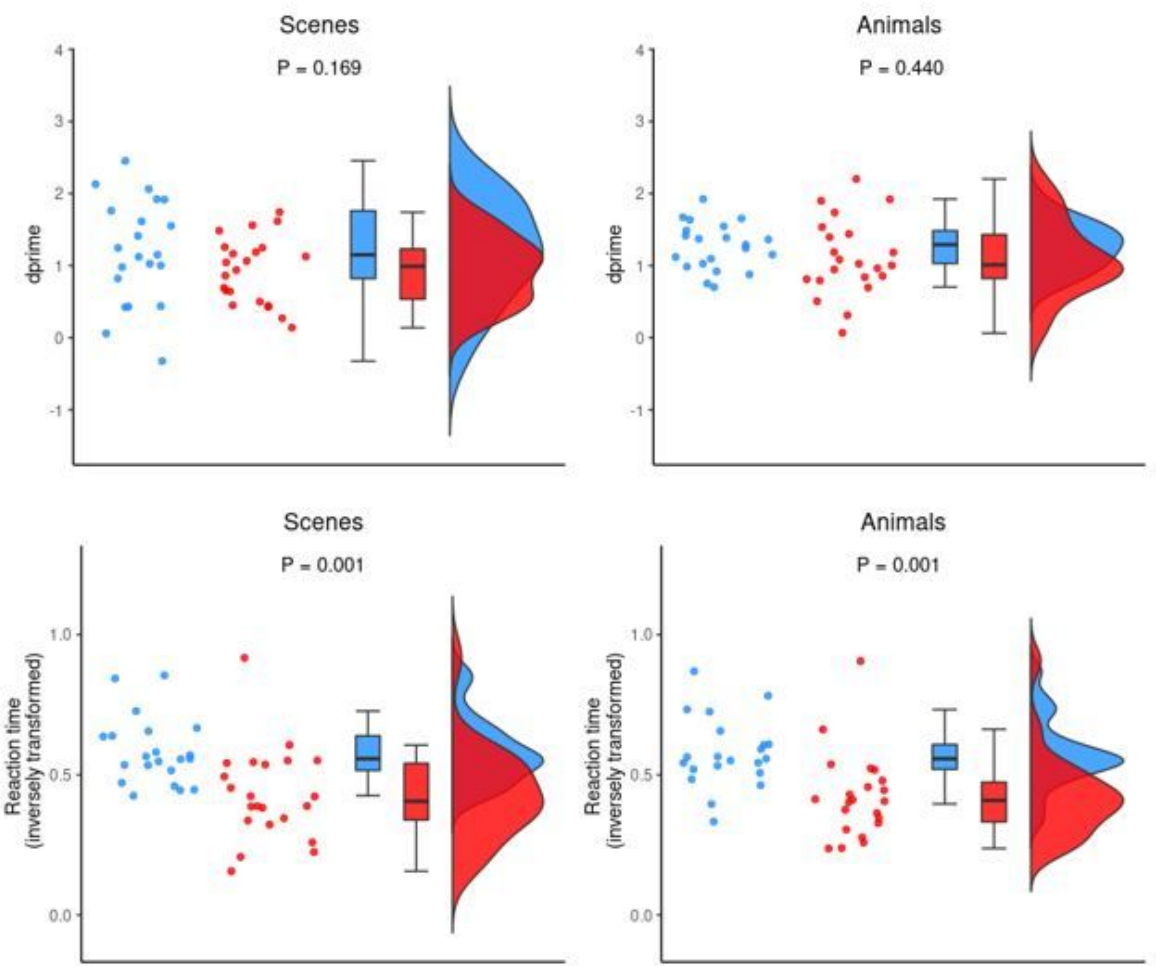

\section{Figure 3}

Behavioural results for the episodic retrieval task. a) Plots representing accuracy according to stimulus category, as measured using dprime (higher values denote better performance). The TBI group had significantly poorer accuracy than healthy controls when retrieving faces $(P=0.037)$. There was a trend for lower accuracy for scenes, although this did not reach statistical significance $(P=0.169)$. There was no significant difference in accuracy for animal stimuli $(P=0.440)$. b) Plots representing reaction time according to stimulus category (note: reaction time was inversely transformed; higher values denote faster performance). As expected, the TBI group was slower than healthy controls when responding to the various stimuli $(P<0.05)$. Note: reported $P$-values were adjusted for multiple comparisons.

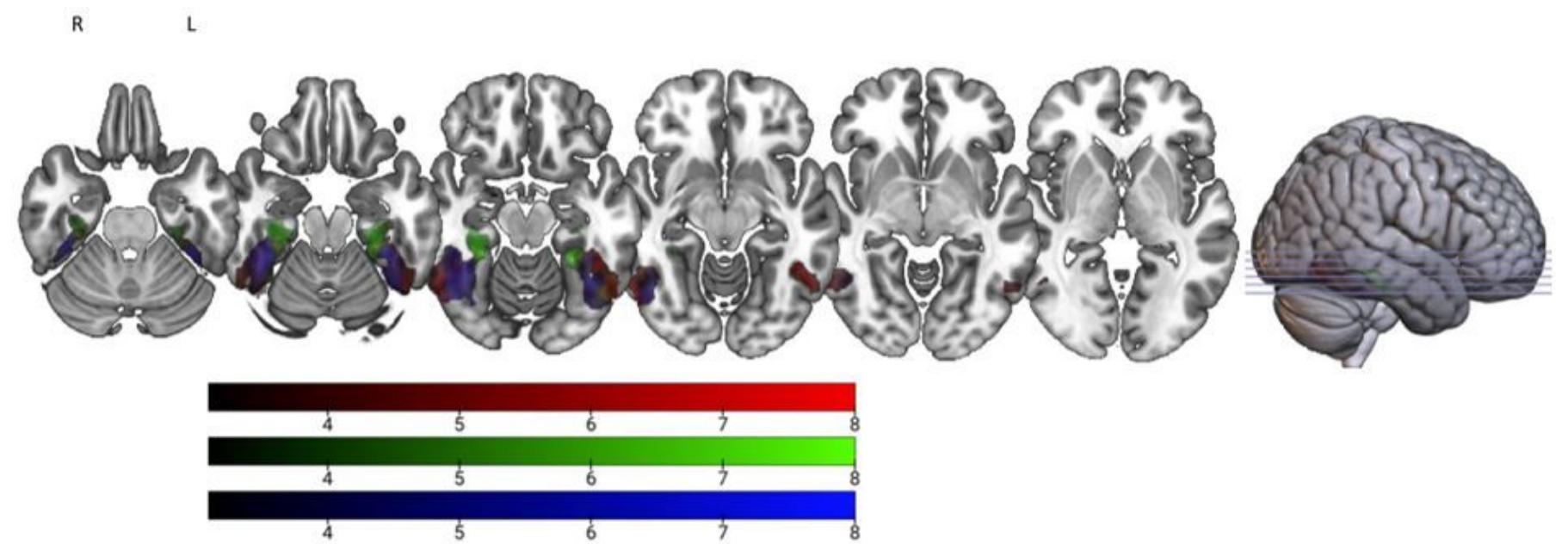




\section{Figure 4}

Overall functional activity elicited during the episodic encoding task for the whole sample. Significant clusters during encoding of faces (blue), scenes (green), and animals (red).
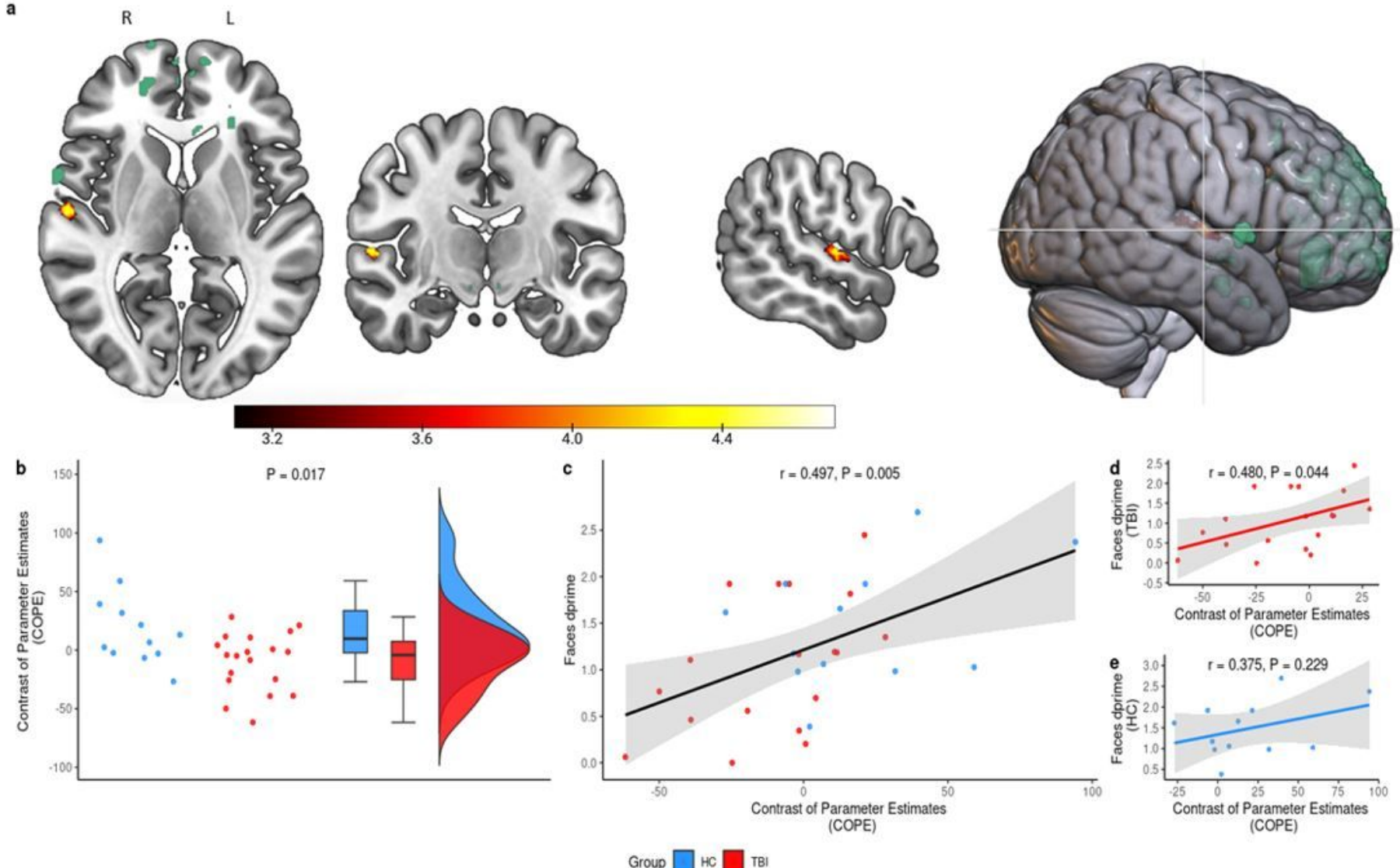

Figure 5

Reduced functional activity during encoding of faces was associated with behavioural performance. a) The TBI group demonstrated a reduced response in the right transverse temporal gyrus extending to the planum temporale during encoding of faces compared to healthy controls. There was no overlap between this cluster and lesions overlap (green). Note: cluster was dilated to increase visibility. b) Plot of the COPE values extracted from the significant cluster in the right transverse temporal gyrus extending to the planum temporale. The difference in COPE values between the TBI group (red) and healthy controls (blue) was significant $(P=0.017)$. c) Overall, there was a significant association between COPE values and the dprime scores for face stimuli $(P=0.005)$. However, further investigation indicated that a significant association was only apparent for $d) \operatorname{TBI}$ group $(P=0.044)$, and not $e)$ healthy controls $(P=0.229)$. 


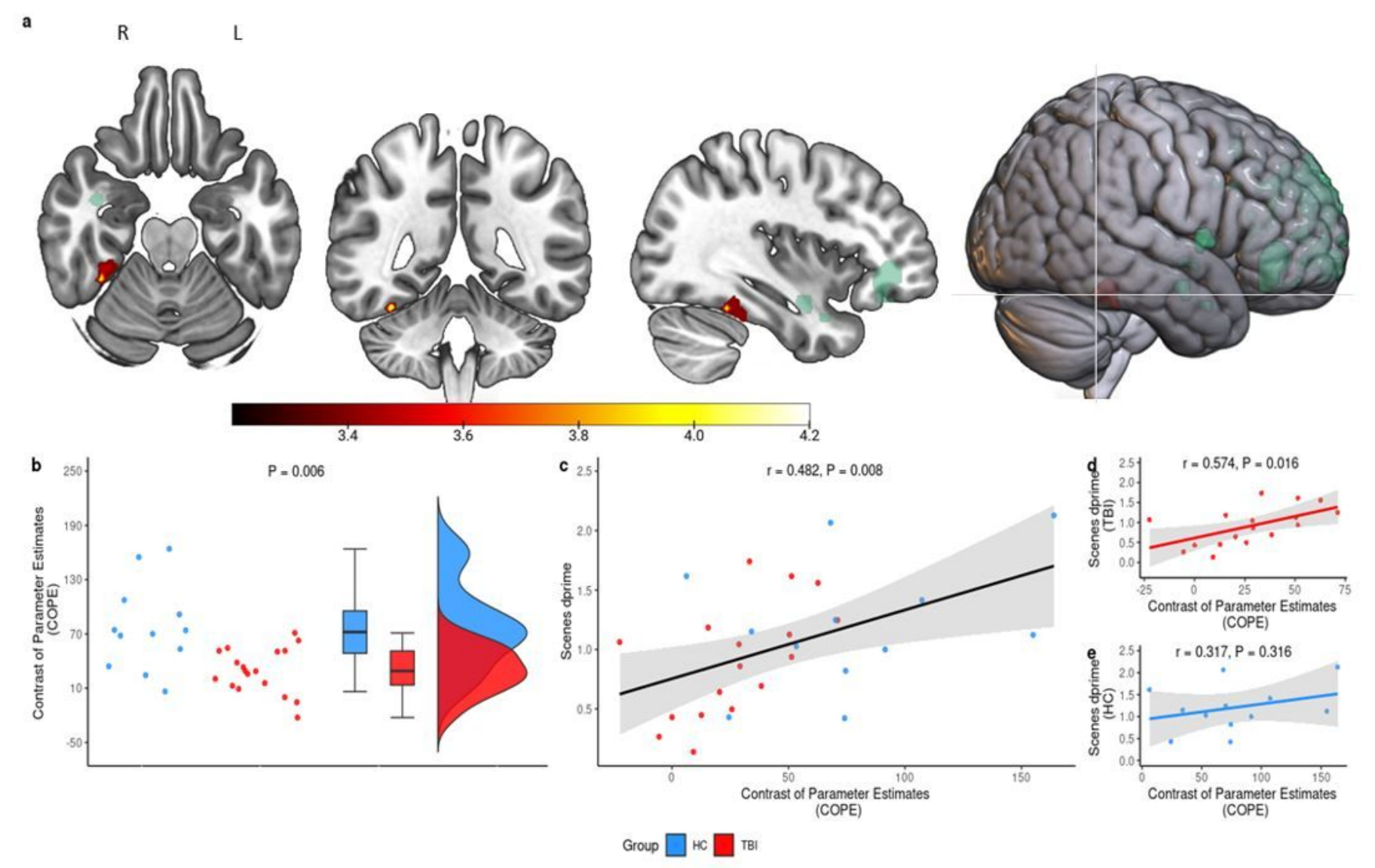

Figure 6

Reduced functional activity during encoding of scenes was associated with behavioural performance. a) In comparison to healthy controls, the TBI group demonstrated reduced activity in the right posterior fusiform gyrus encoding of scene stimuli. There was no overlap between this cluster and lesions overlap (green). Note: cluster was dilated using to increase visibility. b) Plot of the COPE values extracted from the significant cluster in the right posterior fusiform gyrus. The difference in COPE values between the TBI group (red) and healthy controls (blue) was significant $(P=0.006)$. c) Overall, there was a signification association between the COPE values and the dprime for scene stimuli $(P=0.008)$. However, further investigation indicated that a significant association was only apparent for $d)$ TBI group $(P=0.016)$, and not e) healthy controls $(P=0.316)$.

\section{Supplementary Files}

This is a list of supplementary files associated with this preprint. Click to download.

- EpisodicpaperrevisedsupplementaryScientificReports.docx 